CASE REPORT

\title{
Metastatic pancreatic cancer presenting as linitis plastica of the stomach
}

\author{
Shivani Garg, Ramzi Mulki, Daniel Sher
}

Albert Einstein Medical Center, Philadelphia, Pennsylvania, USA

\section{Correspondence to Dr Ramzi Mulki, mulkiram@einstein.edu}

Accepted 25 February 2016

\section{SUMMARY}

Metastatic disease from pancreatic carcinoma involving the stomach is an unusual event, and the pattern of spread in the form of linitis plastica, to our knowledge, has not been reported previously. Local recurrence after curative resection for pancreatic cancer is the most common pattern of disease. We report a case of metastatic pancreatic adenocarcinoma presenting as linitis plastica of the stomach 4 years after curative resection. A 52-year-old man presented with epigastric pain and melaena 4 years after undergoing a Whipple's procedure for a poorly-differentiated pancreatic adenocarcinoma, stage IB; T2NOMO. CT imaging of the abdomen revealed thickening of the gastric wall, and subsequent oesophagogastroduodenoscopy (OGD) revealed diffuse friable erythaematous tissue. The biopsy specimen obtained during the OGD revealed a poorly differentiated adenocarcinoma, with similar appearance to the prior specimen obtained from the pancreas.

\section{BACKGROUND}

Secondary malignant disease involving the stomach is a rare event although autopsy results suggest the incidence of gastric metastasis is higher than clinically reported. This highlights the silent presentation of this unusual metastatic condition and underreporting of gastric metastatic disease. The most common cancers with reported metastasis to the stomach are breast, lung and malignant melanoma. $^{1}{ }^{2}$ While pancreatic adenocarcinoma is known to have local recurrence, haematogenous spread, however, is seen in less than $30 \%$ of patients, with the liver being the most common site. $^{3}{ }^{4}$ Metastatic disease to the stomach has been described in the past as a localised submucosal tumour consistent with pancreatic adenocarcinoma that responded well to gastrectomy. ${ }^{5} 6$ However, metastatic pancreatic adenocarcinoma presenting as linitis plastica of the stomach has not been identified. We describe a case of metastatic pancreatic adenocarcinoma presenting as linitis plastica of the stomach 4 years after curative resection.

\section{CASE PRESENTATION}

A 52-year-old man presented with epigastric pain and melaena for several days. On further questioning, he reported a 30-pound weight loss in the previous 6 months, chronic nausea after food intake, significant for generalised fatigue, and early satiety consistent with gastric outlet obstruction. On physical examination, he had stable vital signs, significant sarcopenia and cachexia, with a body mass index of $16 \mathrm{~kg} / \mathrm{m}^{2}$. He had epigastric tenderness with no active lesions and no bleeding on rectal examination.

He had history of a Whipple's procedure performed for an adenocarcinoma of the pancreas 4 years earlier. The histological diagnosis was a poorly-differentiated adenocarcinoma of the pancreas, (figure 1) stage IB; T2N0M0. ${ }^{7}$ Two years after surgery, the patient presented with weakness and fatigue, which prompted further investigations; he subsequently underwent a CT of the abdomen and pelvis, which showed a $2.4 \times 1.8 \mathrm{~cm}$ mass encasing the superior mesenteric vein $(\mathrm{SMV})$ with persistent mesenteric lymphadenopathy, along with elevation of serum carbohydrate antigen 19-9 level. Biopsy of the mass confirmed presence of malignant cells similar to those of the previous primary pancreatic adenocarcinoma. The patient received combination radiation and chemotherapy of 5-fluorouracil and gemcitabine, which led to a decrease in the size of the mass. He responded well to treatment and was in remission for 2 years, until the current presentation.

\section{INVESTIGATIONS}

The patient's basic laboratory investigations revealed microcytic hypochromic anaemia, mildly elevated transaminases and normal alkaline phosphatase levels. CT imaging of the abdomen and pelvis revealed thickening of the stomach wall when compared to the previous imaging, and an increase in the irregularity of the stomach lining (figures 2 and 3). Furthermore, there was recurrence of the mass encasing the SMV and mesenteric lymphadenopathy.

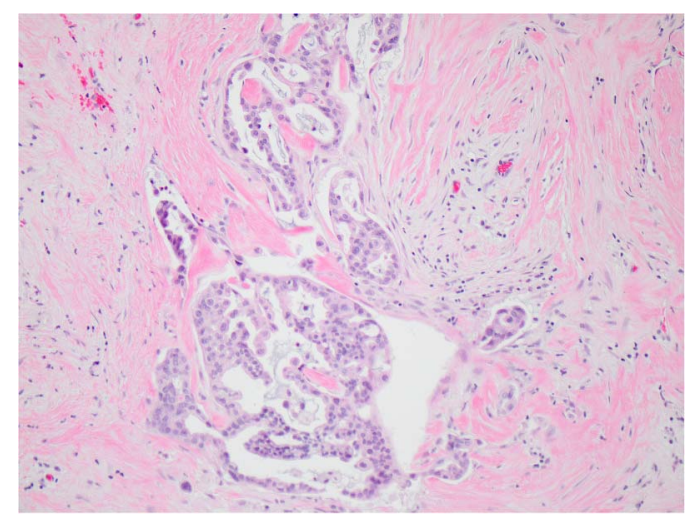

Figure 1 Biopsy section of the pancreas, highlighting the poorly differentiated adenocarcinoma, obtained from the pancreatic tissue at the time of initial diagnosis. 


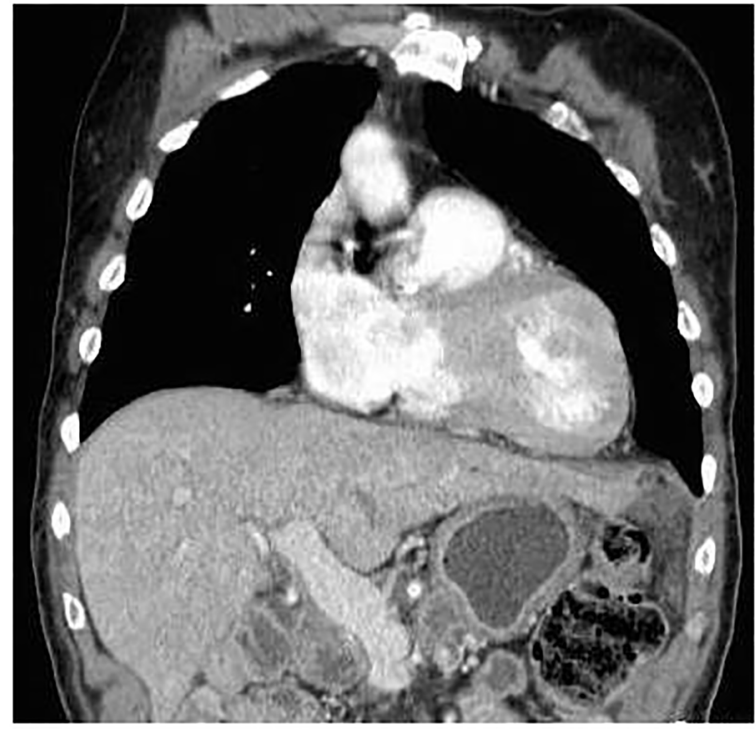

Figure 2 Coronal section CT scan, highlighting the metastatic gastric wall infiltration without local spread.

\section{DIFFERENTIAL DIAGNOSIS}

Based on the above findings, the differential diagnoses included primary gastric cancer, secondary lymphoma after chemotherapy and metastatic pancreatic disease.

\section{TREATMENT}

The patient underwent oesophagogastroduodenoscopy (OGD), which revealed diffuse friable erythaematous tissue in the anterior wall of the stomach, from which multiple biopsies were taken (figure 4). Pathological examination of the tissue specimen revealed invasive adenocarcinoma similar to the primary pancreatic carcinoma (figure 1), with chronic gastritis and intestinal metaplasia (figure 5). Moreover, immunohistochemical studies, using anti-cytokeratin 7 and 20 monoclonal antibodies, showed positivity for cytokeratin 7 and negativity for cytokeratin 20 in the gastric tumour, consistent with the expression pattern in the previously resected pancreatic adenocarcinoma.

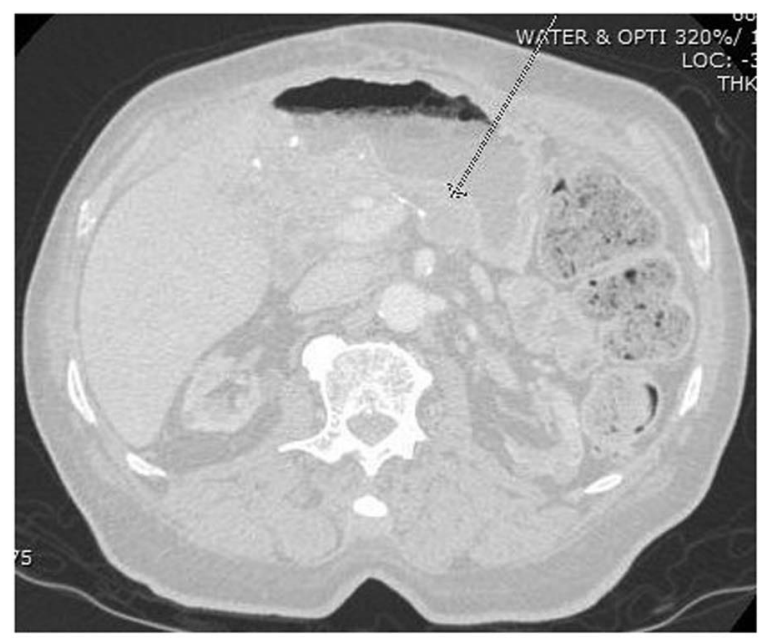

Figure 3 CT scan showing metastatic infiltration of the gastric wall without any locoregional spread.

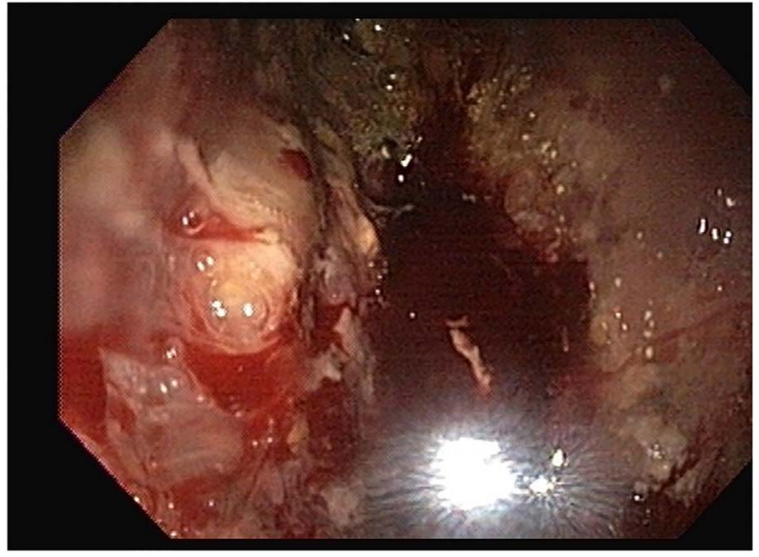

Figure 4 Endoscopic view of the stomach, highlighting the friable erythaematous gastric lining with necrotic and scirrhous changes.

\section{OUTCOME AND FOLLOW-UP}

The diagnosis of recurrent pancreatic adenocarcinoma with metastatic disease presenting as secondary linitis plastica was made. The patient's condition was explained in detail, including the prognosis and limited treatment options. The patient declined palliative chemotherapy and opted for best supportive care.

\section{DISCUSSION}

Pancreatic cancer is the fourth leading cause of death from cancer in men and women, with an average lifetime risk of 1 in $67 .^{8}$ The incidence worldwide of all types of pancreatic cancer (85\% of which are adenocarcinomas) ranges from 1 to 10 cases per 100000 of the population. The 5-year survival rate for all stages of pancreatic cancer has been estimated to be $6 \%$ and is responsible for $7 \%$ of cancer-related deaths. ${ }^{9}$

Pancreatic cancer recurrence after curative resection is common, with reports indicating that most treatment failures are due to local recurrence. ${ }^{4}$ Sperti et $a l^{6}$ evaluated 78 patients who died after curative resection, and revealed that local and hepatic recurrence accounted for $97 \%$ of the total recurrence rate. Haematogenous spread of pancreatic adenocarcinoma to the stomach is extremely rare and most cases are seen during autopsy studies, meaning they remain undetected and under-reported. $^{10}$

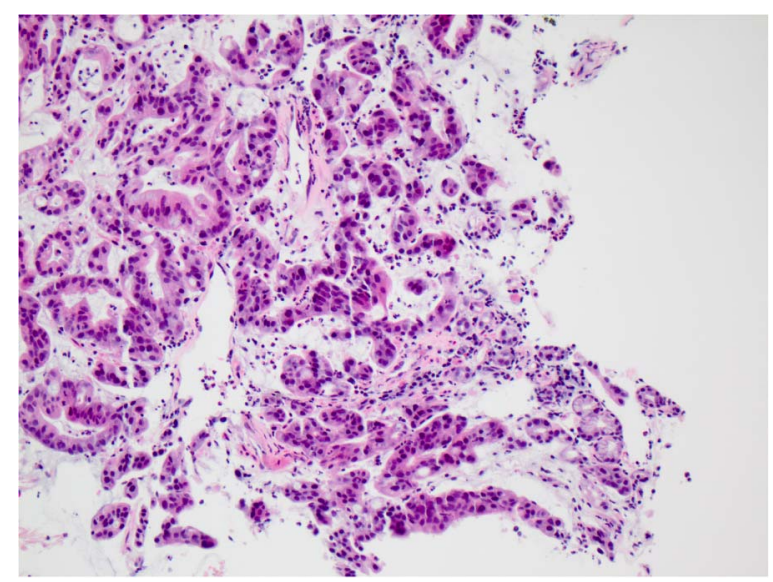

Figure 5 Biopsy section of the stomach, highlighting the poorly differentiated adenocarcinoma, similar to that obtained from the pancreatic tissue at the time of diagnosis. 
A number of pathways for the secondary disease in the gastrointestinal tract by tumour spread have been described. Such pathways include direct invasion from adjacent organs, intraoperative seeding of tumour cells, lymphovascular spread, and intraluminal and intramural spread. ${ }^{11}$ The stomach has a rich lymphovascular supply and therefore haematogenous spread, from carcinoma of the breast, lung and malignant melanoma, commonly presents as gastric linitis plastica, but is rarely reported as one of the common mechanisms involved with spread of pancreatic cancer. In this regard, Willis states that metastasis that occurs by the haematogenous route, leads to the formation of a doughnut-shaped lesion as, initially, it invades the submucosa, which progresses to the development of a circumscribed nodule or plaque that protrudes into the lumen and eventually undergoes central ulceration. ${ }^{8}$

Patients with gastric metastasis often lack specific symptoms, which may explain the reason for the late or underdetection of this pattern of recurrence. These patients are often asymptomatic or present with non-specific symptoms such as abdominal pain, gastrointestinal bleeding, weight loss, anorexia, nausea and vomiting. ${ }^{3}{ }^{10}$ Symptoms such as early satiety and epigastric pain in the setting of a previously resected pancreatic cancer should prompt clinicians to investigate, as they may indicate early presentation of gastric outlet obstruction due to infiltration of stomach lining or due to a mass outside the stomach obliterating its lumen. In advanced cases, clinical examination may be beneficial and the classical signs of succussion splash may be detected. Other rare reported signs include a palpably enlarged stomach, Virchow's node (left supraclavicular lymphadenopathy), Sister Mary Joseph's nodule (periumbilical node), or Blumer's shelf (metastatic tumour felt on rectal examination, with growth in the rectouterine/rectovesical space). ${ }^{12} 13$

Griffin $e t a l^{4}$ reported that CT scanning (with intravenous and oral contrast) is the most frequently used diagnostic modality to detect stomach wall infiltration, local and distant metastasis; followed by ultrasonography of the abdomen and, rarely, scintigraphy. Although CT scanning is the most available high yield diagnostic modality, it has its limitations due to its inability to assess tumour spread to adjacent lymph nodes unless they are significantly enlarged. Moreover, it is not effective in determining the depth of tumour invasion, and cannot reliably support the detection of solitary liver or lung metastases smaller than $5 \mathrm{~mm}$ in diameter. Endoscopic ultrasound is the procedure of choice for locoregional and infiltrative gastrointestinal luminal cancer staging, due to its higher yield in determining the depth of tumour invasion and local lymph node involvement. ${ }^{12-14}$ OGD is a highly sensitive and specific diagnostic test, especially when combined with endoscopic biopsy, and is hence used as a confirmatory test. ${ }^{12-15}$ Davis and Zollinger ${ }^{16}$ suggest that the characteristic appearance of doughnut-shaped friable metastatic masses in the stomach wall are consistent with metastatic melanoma. Other findings observed during endoscopy are calcification or plaques around the lesions, central ulceration, as most of these lesions are friable, and polypoidal masses, however, these findings are non-specific. ${ }^{1}$ Random biopsies beyond lesion areas are also important in achieving a correct tissue diagnosis, as the mucosa is often spared from malignant infiltration, and predominantly involves sub-mucosal and muscular layers. Superficial biopsies can be negative in up to $30 \%$ of patients; therefore, bloc biopsy using the submucosal endoscopic mucosal flap is useful in detecting submucosal tumours. ${ }^{12-14}$ This technique leads to a histopathological diagnosis without any complications, such as haemorrhage or dissemination of tumour.
Collins et $a l^{17}$ followed 210 patients with locally advanced gastric cancers and reported that gastrectomy in the setting of advanced gastric cancer was useful in up to half of patients, with an acceptable perioperative mortality rate. Primary gastric cancers behave differently from metastatic infiltration. The treatment of the underlying primary cancer should be the treatment modality of choice, therefore, the treatment for metastatic gastric linitis plastica or metastatic gastric cancer varies with the underlying primary cancer. ${ }^{17}{ }^{18}$ The treatment modalities in our case were limited due to lack of literature and also absence of complications indicating the need for urgent intervention or surgery. Only one case reported complete cure after resection of gastric tumour, however, the tumour was found in the same place as the lesion detected on preoperative examinations and no other metastatic lesion was detected in the abdomen. ${ }^{4}$

Haematogenous spread of pancreatic cancer to the stomach has previously been reported; ${ }^{5}$ however, to our knowledge, it has not been reported in the literature thus far in the pattern of gastric linitis plastica. Moreover, our case highlights the limited treatment modalities for gastric metastasis from pancreatic adenocarcinoma and the need for further research in this sphere for better management of these patients.

\section{Learning points}

- Haematogenous spread of malignancy to the stomach is atypical, mostly occurring from the breast, lung and malignant melanoma.

- The spread of pancreatic adenocarcinoma to the stomach is extremely rare, and pancreatic cancer causing linitis plastica has not been previously described.

- Bloc biopsies using a submucosal endoscopic mucosal flap may be useful in detecting submucosal tumours, as superficial biopsies may be negative in up to $30 \%$ of patients.

- There are limited treatment modalities available for metastatic pancreatic adenocarcinoma to the stomach, although chemotherapy may be an option.

Contributors SG was the primary author, compiled the manuscript and literature review. RM was the co-author, helped with review of the literature, data collection and compiling the article. DS was the co-author, helped in editing and compiling the articles, and provided the images.

Competing interests None declared.

Patient consent Obtained.

Provenance and peer review Not commissioned; externally peer reviewed.

\section{REFERENCES}

1 Nelson R, Lanza F. Benign and malignant tumors of the stomach (other than carcinoma). Bockus gastroenterology. Philadelphia: WB Saunders, 1985:1255-67.

2 Menuck LS, Amberg JR. Metastatic disease involving the stomach. Am J Dig Dis 1975;20:903-13.

3 Caramella E, Bruneton JN, Roux P, et al. Metastases of the digestive tract. Report of 77 cases and review of the literature. Eur J Radiol 1983;3:331-8.

4 Griffin JF, Smalley SR, Jewell W, et al. Patterns of failure after curative resection of pancreatic carcinoma. Cancer 1990;66:56-61.

5 Takamori H, Kanemitsu K, Tsuji T, et al. Metastatic gastric tumor secondary to pancreatic adenocarcinoma. J Gastroenterol 2005;40:209-12.

6 Sperti C, Pasquali C, Piccoli A, et al. Recurrence after resection for ductal adenocarcinoma of the pancreas. World J Surg 1997;21:195-200.

7 Greene FL. AJCC cancer staging manual. Springer Science \& Business Media, 2002.

8 Jemal A, Siegel R, Xu J, et al. Cancer statistics, 2010. CA Cancer J Clin $2010 ; 60: 277-300$. 
9 American Cancer Society, Pancreatic Cancer [5 January 2016]. http://www.cancer. org/cancer/pancreaticcancer/

10 Green LK. Hematogenous metastases to the stomach. A review of 67 cases. Cancer 1990;65:1596-600.

11 Feczko PJ, Collins DD, Mezwa DG. Metastatic disease involving the gastrointestinal tract. Radiol Clin North Am 1993:31:1359-73.

12 Kobara $\mathrm{H}$, Mori $\mathrm{H}$, Fujihara $\mathrm{S}$, et al. Bloc biopsy by using submucosal endoscopy with a mucosal flap method for gastric subepithelial tumor tissue sampling (with video). Gastrointest Endosc 2013;77:141-5.

13 Kobara H, Mori H, Rafiq K, et al. Evaluation of gastric submucosal tumors using endoscopically visualized features with submucosal endoscopy. Oncol Lett 2014;8:161-8
14 Kobara H, Mori H, Fujiwara S, et al. Bloc biopsy by tunneling method using endoscopic submucosal dissection for an upper gastrointestinal submucosal tumor. Endoscopy 2012;44(Suppl 2 UCTN):E197-8.

15 El-Sourani N, Troja A, Raab HR, et al. Gastric metastasis of malignant melanoma: report of a case and review of available literature. Viszeralmedizin 2014;30:273-5.

16 Davis GH, Zollinger RW. Metastatic melanoma of the stomach. Am J Surg 1960;99:94-6.

17 Collins A, Hatzaras I, Schmidt C, et al. Gastrectomy in advanced gastric cancer effectively palliates symptoms and May improve survival in select patients. J Gastrointest Surg 2014;18:491-6.

18 Hild C, Talha-Vautravers A, Hoefler P, et al. [Metastatic breast cancer to the stomach: an uncommon evolution of breast carcinoma]. Gynecol Obstet Fertil 2014:42:47-50.

Copyright 2016 BMJ Publishing Group. All rights reserved. For permission to reuse any of this content visit http://group.bmj.com/group/rights-licensing/permissions.

BMJ Case Report Fellows may re-use this article for personal use and teaching without any further permission.

Become a Fellow of BMJ Case Reports today and you can:

- Submit as many cases as you like

- Enjoy fast sympathetic peer review and rapid publication of accepted articles

- Access all the published articles

- Re-use any of the published material for personal use and teaching without further permission

For information on Institutional Fellowships contact consortiasales@bmjgroup.com

Visit casereports.bmj.com for more articles like this and to become a Fellow 